\title{
Use of Topical Superoxide Dismutase in the Management of Radiation-induced Fibrosis: A Neglected Opportunity?
}

\author{
Christian Diehl'* \\ ${ }^{*}$ Correspondence: chdiehl@hotmail.com \\ 'Hospital Nacional de Clínicas, Departamento de Dermatología, Facultad de Ciencias Médicas, Universidad Nacional de Córdoba, Santa Rosa 1564, B \\ lberdi - Córdoba - Argentina.
}

\begin{abstract}
Fibrosis of the dermis and keratosis of the epidermis are frequent late complications of irradiation, this being accidental or a consequence of radiotherapy. Fibrosis is a complex tissue response whose predominasnt characteristics are massive deposition of extracellular matrix and excessive fibroblast proliferation. A perpetual cascade of cytokines has been identified post-irradiation in irradiated tissues, and the occurrence of fibrosis is under strong control of the same. Among these increased cytokines, it was clearly demonstrated that TGF-beta1 is a key player. The biological implications of the free radicals generated are now considered important in causing the oxidative damage and hence fibrosis. In such conditions, topical applications of antioxidants could result favorable in the management of radiation-induced fibrosis. Superoxide dismutase (SOD), one of the most potent antioxidants known to date, can be an effective active ingredient at this stage. In the past, various clinical trials have demonstrated the beneficial effects of topical superoxide dismutase on radiation-induced fibrosis. Since then, it seems that the interest for this topical antioxidant has vanished, whilst it still remains an efficient and safe alternative for treating damages caused by irradiation.
\end{abstract}

Background: Radiation-induced fibrosis (RIF) is a complication of radiotherapy that may develop in normal tissue several months to several years following high-dose irradiation. RIF is characterized by non-specific changes in the vascular connective tissue involving excessive extracellular matrix deposition, fibroblast proliferation and the presence of inflammatory infiltrate [45].

Conclusion: Recent progress in understanding the pathophysiology of RIF in conjunction with topical antioxidant therapy suggests a new treatment option for what has long been considered an irreversible process.

\section{Introduction}

Fibrosis of the dermis and keratosis of the epidermis is frequently a delayed complication of irradiation, this being accidental or a consequence of radiotherapy [1]. Dosage, fractionation and duration of irradiation are major factors of fibrotic growth [2]. Hypo-fractionated irradiation has been shown to induce a much higher rate of fibrosis than a conventional fractionation schedule (57\% Vs 16\%) [6]. Fibrosis was commonly observed after seven-month and three-year followups with both simultaneous integrated boost and sequential boost[7]. Comparing accelerated partial breast irradiation (APBI) Vs. conventional external beam radiotherapy, NTCP (Normal Tissue Complication Probability) values appear to be much higher in the latter [8].

Recent studies demonstrate higher rates of occurrence of subcutaneous fibrosis after 15 months [9] (33\%) and after 43 months[10] (44\% Vs $9 \%)$. Additionally, two unrelated studies $[11,12]$ observed $3.2 \%$ of patients developed breast fibrosis 24 months after Intraoperative Radiotherapy with Electrons (ELIOT) during breast conserving surgery, and this rate was shown to reach $33 \%$ after a 48 -month survey. Approximately $5 \%$ of patients developed fibrosis of the entire breast and $27 \%$ around the tumor bed 25 months after IORT using low-energy $\mathrm{X}$-rays [13].

Fibrosis may be related to preexisting collagen diseases [14], concomitant or sequential administration of chemotherapy $[15,16]$, age[17], and the intrinsic radiosensitivity of the connective tissue, which generally varies among patients[18]. After breast-conserving surgery the concurrent use of chemotherapy with radiotherapy is significantly associated with an increased incidence of grade-2 fibrosis[19]. The incidence and prevalence of fibrosis is more common when compared to other radiation-induced morbidities [20].

Fibrosis is a considered a late-stage complication of radiation therapy and might not appear until $5-10$ years post treatment [20].

In another study, the length of time to expression of $90 \%$ of moderate or severe complications was 3.2 years. For subcutaneous fibrosis, the time to reach a specific grade of reaction increases with the grade. This is consistent with the clinical impression that fibrosis progresses in severity over time [21].

\section{Pathophysiology}

Latent radiation damage is characterized by loss of parenchymal cells and excessive formation of fibrous tissue [22]. Fibrosis is a complex tissue response whose predominant characteristics are massive deposition of extracellular matrix and excessive fibroblast proliferation. It is a dynamic process that involves constant tissue remodeling and long-term fibroblast activation. 
Fibrosis is commonly located on skin but has been detected in tissue from the lung, heart and liver [23]. Research has shown that radio-induced fibrosis is an endless scarring process, in which the myofibroblast, a particular type of fibroblast, plays an essential role[1].

The origin of fibroblast activation in fibrosis is debated. Nevertheless, in normal wound healing fibroblasts are transiently activated into myofibroblasts to proliferate and deposit the collagen matrix.

Fibrosis is similar to a wound whereas continuous signals for tissue repair are emitted. These continuous signals can lead to abnormal production of cytokines and growth factors, resulting in chronic, sustained long-term myofibroblast activation leading to fibrosis $[1,2,3]$. Among the various growth factors, it is TGF- $\beta 1$ which orchestrates chronic cell activation and is considered a master switch for this fibrotic mechanism [24].

Tissue homeostasis is maintained by coordinating cell growth and proliferation with the production and turnover of the extracellular matrix. Cells achieve this coordination by constantly signaling to themselves (autocrine activity) and each other (paracrine activity) by means of polypeptides called cytokines (also known as growth factors) [25].

We are beginning to learn that the generation of reactive oxygen species (ROS) by ionizing radiations [26] is a dynamic process involving a number of pro-inflammatory cytokines, profibrotic cytokines and chemokines produced by a variety of cell types, including macrophages, epithelial cells and fibroblasts.

The inflammatory response evoked by the radiation injury results in a stimulative response of tissue cells similar to fibroblasts leading to an increased proliferation and differentiation as well as collagen secretion $[17,23]$.

The cell type PMF VI is the terminally differentiated end cell of the fibroblast stem cell system. PMF VI is characterized by a specific capacity for the synthesis of interstitial collagens types I, III and V, proteoglycans and other extracellular matrix components [28]. Ionizing radiation has been shown to induce premature terminal differentiation in the fibroblast/fibrocyte cell system, presumably by a significant depletion of mitotically active progenitor fibroblasts due to an induced transition into post mitotic fibrocytes. For the radiation-induced post mitotic fibrocytes, it has been demonstrated that synthesis of interstitial collagens types I, III and V is enhanced by a factor of 5-8 as compared to progenitor fibroblast populations [28].

Recent studies also suggest that radiation-induced fibrosis is a complex process involving the initiation and prolongation of fibrogenic processes through an intercommunication of different cell types. This communication appears to be mediated by the production of specific cytokines and growth factors. An immediate and transient expression of growth factors, like platelet derived growth factor (PDGF), IL-1, TGF- $\beta$ and TNF-á occurs within hours of irradiation [23]. This altered expression of growth factors and cytokines may result in the modulation of the cellular interactions of cell types involved in fibrotic reactions.
A perpetual cascade of cytokines has been identified post irradiation in lung tissues, beginning immediately at the time of irradiation and persisting up to the appearance of the latent injury [15]. In the efferent limb or at the target cell, (fibroblast) growth factor receptors are activated, leading to signal transduction. The stimulated fibroblasts respond by turning on collagen genes, leading to the production of extracellular matrix proteins [29]. It has been postulated these early alterations, identified within days to weeks post-irradiation, persist and play a major role in the subsequent development of chronic fibrosis.

The presence of progressive hypoxia has also been noted after irradiation of the lung and spinal cord [30, 31]. Because hypoxia itself is known to generate reactive oxygen species, promote inflammation and vascular damage, activate profibrotic cytokines and promote collagen formation [32, 33, 34], it has been suggested that post-irradiation hypoxia may be an important contributor to maintenance of the injured phenotype [35].

\section{Key role of TGF- $\beta 1$}

Studies involving the immunohistochemical staining of TGF- $\beta$ in irradiated lung tissue demonstrates an acute and long lasting increase in the expression of TGF- $\beta$ in lung tissue following irradiation [36]. The most striking increase in TGF- $\beta$ immunoreactivity was seen at the beginning of the pneumonic phase (two and four weeks post-irradiation). At this peak of TGF- $\beta$ expression, inflammatory cells constituted the majority of cells expressing TGF- $\beta$.

Cytokines regulate all aspects of tissue remodeling. TGF- $\beta$ can now be considered the prototype of a multifunctional cytokine, especially after the initial discovery that it could act both as an inhibitor and as a stimulator of cell replication. Additionally, it acts to control the synthesis of many components of the extracellular matrix $[37,38]$.

The TGF- $\beta 1$ gene is upregulated in response to tissue injury, and TGF- $\beta 1$ is the isoform most implicated in fibrosis [25]. The effects of TGF- $\beta$ on the synthesis and definition of extracellular matrix are mediated by the type I receptor, while the effects on cell growth and proliferation are mediated by the type II receptor [25]. Other cytokines involved with TGF- $\beta 1$ in tissue remodeling after injury include platelet derived growth factor (PDGF), basic fibroblast growth factor (bFGF), tumor necrosis factor (TNF) and interleukin 1 (IL-1). All of these stimulate cell proliferation and migration and/or formation of new vessels and/or promote inflammation, but TGF- $\beta 1$ is unique in its widespread actions that enhance the deposition of extracellular matrix [38].

It has been shown that extrinsic TGF- $\beta$ could stimulate the formation of connective tissue and collagen [39], and subcutaneous injection of TGF- $\beta$ in newborn mice induces angiogenesis and causes rapid activation of fibroblasts to produce collagen. The new tissue formed is essentially granulation tissue, resembling that discovered during physiological wound repair. Furthermore, it was shown that TGF- $\beta$ in vitro had stimulatory effects on the formation of collagen by a variety of rodent and human fibroblasts. Moreover, 
it was shown that specific antibodies to TGF- $\beta$ could partially abolish this stimulatory effect.

TGF- $\beta 1$ also inhibits the functioning of T cells and B cells and their production of TNF and IL-1 [40]. Whereas TGF- $\beta 1$ can function as either an agonist or an antagonist of cell proliferation and in flammation, it consistently and potently acts on cells to induce the deposition of extracellular matrix. TGF- $\beta 1$ causes the deposition of extracellular matrix by simultaneously stimulating cells to increase several-fold the synthesis of most matrix proteins, decrease the production of matrix-degrading proteases, increase the production of inhibitors of these proteases and modulate the expression of integrins in a manner that increases cellular adhesion to the matrix [25]. The accumulation of matrix in tissues is the chief pathologic feature in fibrotic diseases.

In both animal and humans, acute, limited injury is accompanied by only a transient increase in TGF- $\beta 1$, and fibrosis does not occur. With repeated injuries, the increase in TGF- $\beta 1$ production is sustained, leading to the progressive deposition of extracellular matrix in tissue fibrosis [41]. Repeated injury, with continued autoinduction of TGF- $\beta 1$, overrides the normal termination signals, creating a chronic, vicious circle of TGF- $\beta 1$ overproduction.

Thus, TGF- $\beta 1$ is a double-edged sword with both therapeutic and pathological potential [42]. There is a delicate balance between TGF- $\beta 1$ 's beneficial actions in normal tissue repair and its fibrogenic potential, and antagonizing the actions of TGF- $\beta 1$ might be beneficial in a number of human diseases [43].

There are clearly situations in which persistent over-expression or deregulated activation of TGF- $\beta$ elicits an over-response which may lead to serious fibrotic or proliferative disease [38]. Apart from being a local growth factor, TGF- $\beta 1$ has also systemic effects, such as cachexia and multiple fibrosis [44].

Many studies have clearly demonstrated that TGF- $\beta 1$ is a key player in fibrosis. Studies in pig models showed that TGF- $\beta$ was detected in skin at 6 hours after $\gamma$-irradiation at both the protein and messenger RNA levels, for single doses of radiation ranging from 16 to $64 \mathrm{~Gy}$ [45]. It was also demonstrated that TGF- $\beta 1$ was overexpressed in a pig skin model of fibrosis throughout all the phases of fibrosis development [45]. The TGF- $\beta 1$ mRNA level was increased 12 -fold in the irradiated skin during the early erythematous phase, which started three weeks after irradiation. During the later phases of fibrosis, from 6 to 12 months after irradiation it remains 10-fold elevated over the basal level in the repaired skin [45].

Several concepts align when comparing the general functions of TGF- $\beta 1$ and its role in fibrosis. Its bimodal action on cell proliferation, with inhibition of epithelial cells and activation of fibroblasts, can certainly favor fibrosis and scar development. Similarly, the capacity of TGF- $\beta$ to induce apoptosis in specific cell types can favor parenchymal damage and replacement by fibrotic tissue [42].

The influence of cytokines on extracellular superoxide dismutase (EC-SOD) expression by human dermal fibroblasts has been investigated [46]. EC-SOD is primarily a tissue enzyme, and forms the major physiological defense against superoxide radicals released to the extracellular space. Expression of EC-SOD occurs in only a few cell types, and fibroblasts are an important source of EC-SOD in the body [47].

Expression of EC-SOD is profoundly influenced by cytokines involved in the inflammatory response. Whereas IFN- $\gamma$, TNF-á and IL-1 enhance EC-SOD production, IFN-á, IL-6 and PgE2 do not influence EC-SOD expression, but TGF- $\beta$ markedly progressively depressed EC-SOD expression down to about 3-4\% of controls. It was also noticed that TGF- $\beta$, which depresses superoxide production, depressed EC-SOD expression in itself but also depressed the stimulatory effects of IFN- $\gamma$ and IL-1[47].

\section{Radiotherapy, free radicals (ROS) and the development of ra-} diation fibrosis:

Radiation may act with other atoms or molecules in the cells (particularly water) to produce free radicals that are able to diffuse far enough to reach and damage critical targets [26]. The free radicals generated cause oxidative damage and hence fibrosis [42]. Many chemotherapeutic agents (especially bleomycin) may potentiate the damaging effects of radiation and are most likely linked to the production of ROS and redox cycling [42]. Therefore, ROS may play a synergistic effect during the combined radiotherapy and chemotherapy regimens to potentiate the damaging effects.

The free radicals are formed as a result of direct damage to the cellular molecules of water by ionization:

$$
\begin{aligned}
& 2 \mathrm{H}_{2} \mathrm{O} \rightarrow \mathrm{H}_{2} \mathrm{O}^{*+}+\mathrm{e} \neg+\mathrm{H}_{2} \mathrm{O}^{*} \\
& \mathrm{H}_{2} \mathrm{O}^{*} \rightarrow \mathrm{H}^{*}+\mathrm{OH}^{*} \text { (hydroxyl radical) } \\
& \mathrm{H}_{2} \mathrm{O}^{*+}+\mathrm{H}_{2} \mathrm{O} \rightarrow \mathrm{H}_{3} \mathrm{O}^{+}+\mathrm{OH}^{*} \\
& \mathrm{OH}^{*}+\mathrm{OH}^{*} \rightarrow \mathrm{H}_{2} \mathrm{O}_{2} \text { (hydrogen peroxide) } \\
& \mathrm{O}_{2}+\mathrm{e} \neg->\mathrm{O}^{2-} \text { (superoxide radicals) } \\
& 2 \mathrm{O}^{2-}+2 \mathrm{H}^{+}->2 \mathrm{H}_{2} \mathrm{O}_{2} \text { (hydrogen peroxide) }
\end{aligned}
$$

It is now understood that the interaction of ionizing radiation with living tissue causes the production of free radicals. These free radicals play a primary role in initiating the biological damage and the ensuing secondary inflammatory response [42].

The tissue damage induces directional migration of neutrophils and macrophages into the extra vascular parenchymal tissue and initiates fibroblast recruitment and proliferation. Extra vascular neutrophils and macrophages are stimulated by contact with degraded collagen to release free radicals and proteases. This second wave of free radicals might play a central role in the formation and extension of fibrotic tissue in a self-maintained cycle [42].

After radiation therapy, oxidative stress is present at 15-20 weeks after initial exposure, which correlates with the delayed clinical onset of radiation-induced damage. Studies [48] show that ROS such as superoxide anion and hydrogen peroxide, at submicromolar lev- 
els, appear to act as a novel intra- and intercellular "messenger" capable of promoting growth responses in culture. The mechanisms may involve direct interaction with specific receptors or oxidation of growth signal transduction molecules such as protein kinases, protein phosphatases, transcription factors and transcription factor inhibitors. It is also possible that hydrogen peroxide may modulate the redox state and activity of these important signal transduction proteins through changes in cellular levels of GSH and GSSG.

Critical balances appear to exist in the relationship between cell proliferation and lipid peroxidation in cell death. Progression to a substantially pro-oxidant state, while initially leading to enhanced proliferative responses, results subsequently in increased cell death.

Moreover, an increasing bulk of experimental and clinical data supports a contributory role of oxidative stress in the pathogenesis of fibrogenesis. Indeed, many etiological agents of fibrogenesis stimulate free radical reactions either directly or through inflammatory stimuli. Free radicals, as well as products of their reaction with biomolecules, appear to modulate the activity of the two cellular types mainly involved in the process, namely phagocytes and extracellular matrix-producing cells.

Lipid peroxidation and certain lipid peroxidation products induce genetic over-expression of fibrogenic cytokines, the key molecules in the pathomechanisms of fibrosis, as well as increased transcription and synthesis of collagen. Both these events can be down-regulated, at least in experimental models, by the use of antioxidants. The effect of oxidative stress on cytokine gene expression appears to be an important mechanism by which it promotes connective tissue deposition [49].

Therefore, supplying SOD supplements could directly and/or indirectly enhance the cells natural antioxidant defenses and reduce oxidative stress at cellular and tissue levels, and inhibit the process of fibrogenesis.

\section{Superoxide Dismutase (SOD):}

Since the discovery of SOD it has become clearer that these enzymes provide an essential defense against the superoxide radical. In the presence of SOD, superoxide is dismutated to $\mathrm{H}_{2} \mathrm{O}_{2}$ and $\mathrm{O}_{2}$. $\mathrm{H}^{2} \mathrm{O}^{2}$ is then subsequently eliminated by catalase.

$\mathrm{O}^{2-}+\mathrm{O}^{2-}$ superoxide dismutase $>\mathrm{O}_{2}+\mathrm{H}_{2} \mathrm{O}_{2}$

$2 \mathrm{H}_{2} \mathrm{O}_{2}$ catalase $>\mathrm{O}_{2}+2 \mathrm{H}_{2} \mathrm{O}$

In 1994, investigators studied the therapeutic effects of SOD in a Polyethylene glycol ointment (PEG-SOD) administrated twice a day for three months on radiation-induced fibrosis [51]. After 6 months, a $41 \%$ reduction was observed compared to pre-treatment. The therapeutic effect was greater on the most recent onset of fibrosis and there was a chronological order to the different recovery stages. After 6 weeks pain was reduced or disappeared. After 3 months it was observed the fibrous texture started to soften and breakup. An effective reduction of the surface as well as pigment lightening did not usually occur until the $4^{\text {th }}$ month. This major discovery suggests that radiation-induced fibrosis may be reversible, and antioxidant compounds may be effective in the treatment of fibrosis [1].

The development of a standardized pig model of RIF provided an excellent tool to show the effects of SOD in fibrosis [52]. Strikingly, the $70 \%$ decrease in the cutaneous surface area and volume of the fibrotic block were highly significant compared to the clinical and autopsy groups for the control animals.

It was further demonstrated [53] that over-expression of a transgene for human Mn/SOD decreased the latent effects of whole lung irradiation. In transgenic mice, over-expression of EC-SOD was found to confer protection against the radiation-induced lung injury, with a corresponding decline in oxidative stress [54].

Similarly, a novel compound with SOD mimetic properties was shown to protect lung tissue from radiation-induced injury [55]. Interestingly, there was a significant reduction in the plasma level of the TGF- $\beta$ in the group of mice treated with this compound.

In another study [56] a deficiency of endogenous MnSOD, considered to favor cell proliferation, was observed in cultured radiation-induced fibrosis (RIF) cells. Exposure to $\mathrm{Cu} / \mathrm{Zn}$ SOD enhanced endogenous MnSOD activity and protein levels, and significantly reduced TIMP and TGF- $\beta 1$ gene expression in RIF cells, making modulation of RIF skin fibroblasts effective via indirect endogenous MnSOD activation. TIMP reduction accounted for the elimination of collagenase activity inhibition and the subsequent digestion of excess extracellular matrix deposition, as well as RIF reversibility in vivo.

One study [3] clearly demonstrated that SOD did not induce myofibroblast cell death, whereas it significantly reduced TGF- $\beta 1$ expression. This suggests that SOD might serve as a potent antagonist of this major fibrogenic growth factor. This study also showed that SOD antifibrotic action occurred in vitro through the reversion of myofibroblasts into normal fibroblasts.

\section{Review of topical SOD in radio-induced fibrosis:}

In 1996, a study conducted at Institut Curie in Paris [57] reported the treatment of 42 patients presenting with clinically evaluable cutaneous fibrosis after radiotherapy for breast carcinoma. The time elapsed between irradiation and treatment ranged from three months to forty years (mean delay + SD: $8.5+8.4$ years). $\mathrm{Pa}$ tients were treated for three months with an SOD topical preparation, and sequential cutaneous punch biopsies were performed before and three months after completion of the treatment. Histochemical grading, using an objective spectrophotometric method, showed a decrease in fibrosis in $74 \%$ of patients treated.

In 2004, the anti-fibrotic effect of an SOD ointment applied BID for 90 days was evaluated in 44 patients with clinical radiofibrosis following conservative treatment of breast cancer [58]. The ointment was found to be effective in reducing radiation-induced fibrosis by lowering the pain score in $90 \%$ of patients and a decrease of the fibrotic area size in $50 \%$ of the cases after six months. 
Mammography suggested decreased fibrosis in one third of patients, while thermography showed that it was decreased in $80 \%$ of patients. Clinical changes persisted throughout the study, and tolerance was reported as excellent with $92 \%$ of patients reporting a greater degree of local comfort.

\section{Conclusion}

Why did interest diminish for this apparently effective RIF treatment? Perhaps it was because SOD is mainly extracted from bovine tissues. The epidemic of bovine spongiform encephalitis (BSE) has raised safety concerns about bovine extracts, which may partly explain the decline.

Currently, there are only a few topical products whose composition contains significant amounts of SOD, including products containing SOD derived from non-mamalian (vegetal) sources. The few studies published covering the management of RIF by topical SOD show favorable results with a decrease in pain, fibrotic area, and fibrotic density (Figure 1).

In conclusion, it is suggested that topical SOD may represent a safe and effective addition to our armamentarium for the management of RIF, although further clinical trials are clearly warranted.

\section{List of abreviations}

APBI-Accelerated partial breast irradiation : BSE- Bovine spongiform encephalopathy : bFGF-Basic fibroblast growth factor : EC-SOD-Extracellular superoxide dismutase : GSH-Glutathione : IFN-Interferon : IL-Interleukin : IORT-Intraoperative radiotherapy : NTCP-Normal tissue complication probability : PDGF-Platelet-derived growth factor: PEG-Polyethylene glycol : PgE2-Prostaglandin E2 : PMF-Postmitotic fibrocyte : RIF-Radiation-induced fibrosis : RNA-Ribonucleic acid : ROS-Reactive oxygen species : SOD-Superoxide dismutase :
TGF-Transforming growth factor: TNF-Tumor necrosis factor : TIMPTissue inhibitor of metalloproteinase.

\section{Article History}

Editor: Najmunnisa Nasreen, University of Florida, USA.

EIC: G.J. Peters, VU University Medical Center, Netherlands. Received: 16-Dec-2011 Revised: 01-Feb-2012

Accepted: 14-Feb-2012 Published: 20-Mar-2012

\section{References}

1. Anonymous. Effects of ionizing radiation on human skin. Clefs CEA, n०48, 2003

2. Calle, R. et al. Conservative management of operable breast cancer: ten years experience at the Foundation Curie. Cancer 42, 2045-2053

3. Habibollahi, F. et al. Assessment of skin dose and its relation to cosmesis in the conservative treatment of early breast cancer. Int J Radiat Oncol Biol Phys 14, 291-296

4. Levit, S. H. et al.. In: Principles and practice of radiation oncology. Breast Cancer p730-792

5. Borger, J. H. et al. Dose and volume effects on fibrosis after breast conservation therapy. Int J Radiat Oncol Biol Phys 30, 1073-1081

6. Fehlauer, F. et al. Late effects and cosmetic results of conventional versus hypofractionated irradiation in breast-conserving therapy. Strahlenther Onkol 181, 625-631

7. Raiyawa, T. et al. Late effects and cosmetic results of simultaneous integrated boost versus sequential boost after conventional irradiation in breast-conserving therapy; out come of 7 months follow-up. J Med Assoc Thai 92, 390-397

8. Jothy Basu, K. S. et al. Normal tissue complication probability of fibrosis in radiotherapy of breast cancer: accelerated partial breast irradiation vs conventional external-beam radiotherapy. $J$ Cancer Res Ther 4, 126-130

9. Hepel, J. T. et al. Toxicity of three-dimensional conformal radiotherapy for accelerated partial breast irradiation. Int $J$ Radiat Oncol Biol Phys 75, 1290-1296

10. Wadasadawala, T. et al. Accelerated partial-breast irradiation
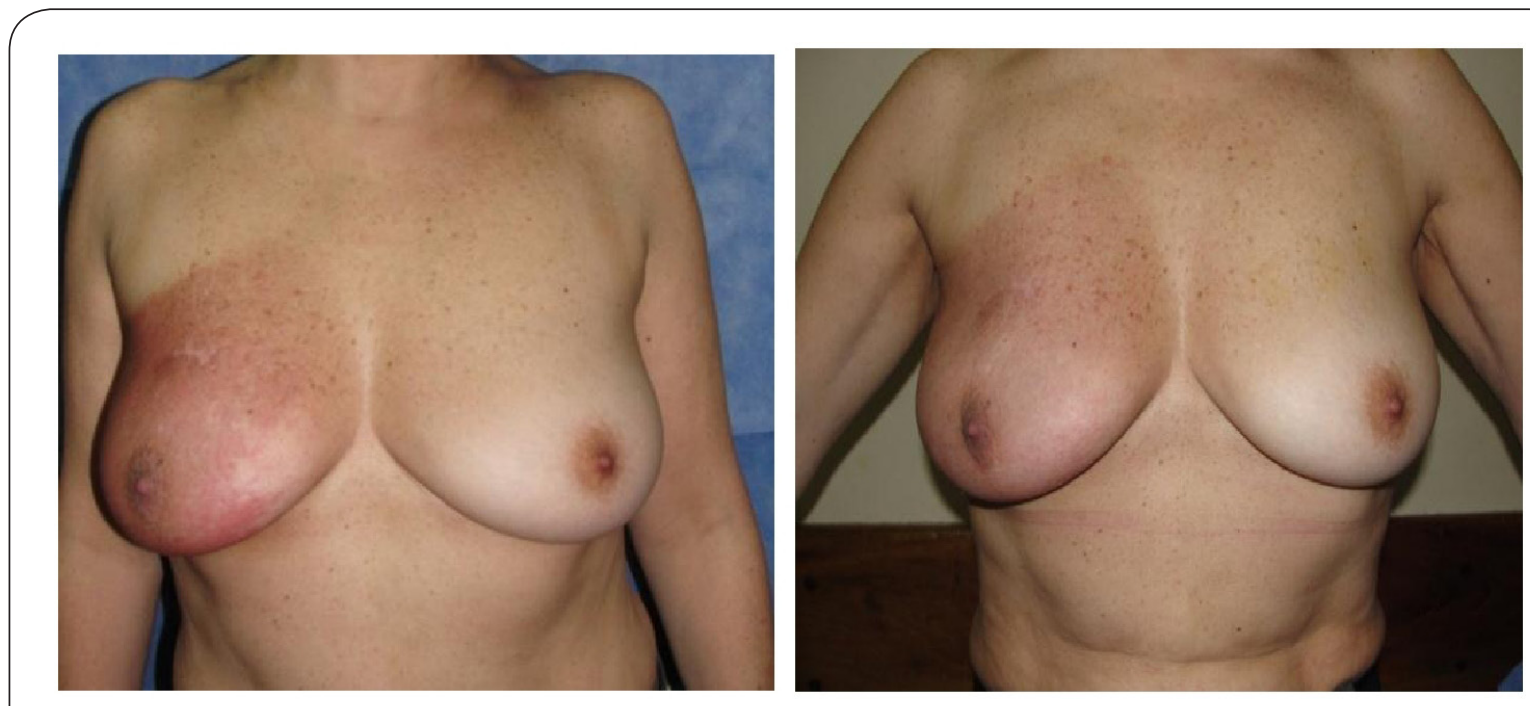

Figure.1: Patient at D0 and D12 of treatment with topical SOD (courtesy Dr. Portas) 
vs conventional whole-breast radiotherapy in early breast cancer: a case-control study of disease control, cosmesis, and complications. J Cancer Res Ther 5, 93-101

11. Veronesi, U. et al. Full-dose intraoperative radiotherapy with electrons during breast-conserving surgery: experience with $\mathbf{5 9 0}$ cases. Ann Surg 242, 101-106

12. Mussari, S. et al. Full-dose intraoperative radiotherapy with electrons in breast cancer. First report on late toxicity and cosmetic results from a single-institution experience. Strahlenther Onkol 182, 589-595

13. Kraus-Tiefenbacher, U. et al. Long-term toxicity of an intraoperative radiotherapy boost using low energy $\mathrm{X}$-rays during breastconserving surgery. Int J Radiat Oncol Biol Phys 66, 377-381

14. Fleck, R. et al. Consequences of breast irradiation in patients with pre-existing collagen vascular diseases. Int J Radiat Oncol Biol Phys 17, 829-833

15. Rubin, P. et al. A perpetual cascade of cytokines postirradiation leads to pulmonary fibrosis. Int J Radiat Oncol Biol Phys 33, 99-109

16. Rube, C. E. et al. Dose-dependent induction of transforming growth factor beta (TGF-beta) in the lung tissue of fibrosis-prone mice after thoracic irradiation. Int J Radiat Oncol Biol Phys 47, 10331042

17. Lilla, C. et al. Predictive factors for late normal tissue complications following radiotherapy for breast cancer. Breast Cancer Res Treat 106, 143-150

18. Turesson, I. Individual variation and dose dependency in the progression rate of skin telangiectasia. Int J Radiat Oncol Biol Phys 19, $1569-1574$

19. Toledano, A. et al. Concurrent administration of adjuvant chemotherapy and radiotherapy after breast-conserving surgery enhances late toxicities: long-term results of the ARCOSEIN multicenter randomized study. Int J Radiat Oncol Biol Phys 65, 324332

20. Roberts, A. B. et al. Transforming growth factor type beta: rapid induction of fibrosis and angiogenesis in vivo and stimulation of collagen formation in vitro. Proc Natl Acad Sci U S A 83, 4167-4171

21. Brandes, M. E. et al. Transforming growth factor beta 1 suppresses acute and chronic arthritis in experimental animals. J Clin Invest 87, 1108-1113

22. Fajardo, L. F. et al. Morphology of radiation effects on normal tissues, in Perez CA, Brady LW (eds): Principles and practice of radiation oncology (ed 2), Philadelphia, PA, JB Lippincott Company pp114-123

23. Border, W. A. et al. Fibrosis linked to TGF-beta in yet another disease. J Clin Invest 96, 655-656

24. Moses, H. L. et al. Transforming growth factor production by chemically transformed cells. Cancer Res 41, 2842-2848

25. Border, W. A. et al. Transforming growth factor beta in tissue fibrosis. N Engl J Med 331, 1286-1292

26. Riley, P. A. Free radicals in biology: oxidative stress and the effects of ionizing radiation. Int $J$ Radiat Biol 65, 27-33

27. Anscher, M. S. The irreversibility of radiation-induced fibrosis: fact or folklore? J Clin Oncol 23, 8551-8552

28. Rodemann, H. P. et al. Cellular basis of radiation-induced fibrosis. Radiother Oncol 35, 83-90

29. Wazer, D. E. et al. Factors influencing cosmetic outcome and complication risk after conservative surgery and radiotherapy for early-stage breast carcinoma. J Clin Oncol 10, 356-363

30. Vujaskovic, Z. et al. Radiation-induced hypoxia may perpetuate late normal tissue injury. Int J Radiat Oncol Biol Phys 50, 851-855

31. Li, Y. Q. et al. Hypoxia in radiation-induced blood-spinal cord barrier breakdown. Cancer Res 61, 3348-3354

32. Shweiki, D. et al. Vascular endothelial growth factor induced by hypoxia may mediate hypoxia-initiated angiogenesis. Nature 359, 843-845

33. Haroon, Z. A. et al. Early wound healing exhibits cytokine surge without evidence of hypoxia. Ann Surg 231, 137-147

34. Zhong, Z. et al. Cyclosporin A increases hypoxia and free radical production in rat kidneys: prevention by dietary glycine. Am J Physiol 275, F595-604

35. Anscher, M. S. et al. Recent progress in defining mechanisms and potential targets for prevention of normal tissue injury after radiation therapy. Int J Radiat Oncol Biol Phys 62, 255-259

36. Beadle, G. F. et al. The effect of adjuvant chemotherapy on the cosmetic results after primary radiation treatment for early stage breast cancer. Int J Radiat Oncol Biol Phys 10, 2131-2137

37. Massagué J. Transforming growth factor á. J Biol. Chem. 265, 21393-

38. Sporn, M. B. et al. Transforming growth factor-beta: recent progress and new challenges. J Cell Biol 119, 1017-1021

39. Johansson, S. et al. Dose response and latency for radiationinduced fibrosis, edema, and neuropathy in breast cancer patients. Int J Radiat Oncol Biol Phys 52, 1207-1219

40. Bentzen, S. M. et al. Latent-time estimation for late cutaneous and subcutaneous radiation reactions in a single-follow-up clinical study. Radiother Oncol 15, 267-274

41. Yamamoto, T. et al. Sustained expression of TGF-beta 1 underlies development of progressive kidney fibrosis. Kidney Int 45, 916-927

42. Hamsakutty H. Radiation-induced fibrosis. University of lowa

43. Bernasconi, P. et al. Expression of transforming growth factorbeta 1 in dystrophic patient muscles correlates with fibrosis. Pathogenetic role of a fibrogenic cytokine. J Clin Invest 96, 11371144

44. Zugmaier, G. et al. Transforming growth factor beta $\mathbf{1}$ induces cachexia and systemic fibrosis without an antitumor effect in nude mice. Cancer Res 51, 3590-3594

45. Martin, M. et al. TGF-beta1 and radiation fibrosis: a master switch and a specific therapeutic target? Int J Radiat Oncol Biol Phys 47 277-290

46. Marklund, S. L. Regulation by cytokines of extracellular superoxide dismutase and other superoxide dismutase isoenzymes in fibroblasts. J Biol Chem 267, 6696-6701

47. Marklundt S. Biochem J 266, 213-9

48. Burdon, R. H. Superoxide and hydrogen peroxide in relation to mammalian cells proliferation. Free Radic. Biol. Med 18, 775-794

49. Poli, G. et al. Oxidative damage and fibrogenesis. Free Radic. Biol. Med 22, 287-305

50. Xavier, S. et al. Amelioration of radio-induced fibrosis: inhibition of transforming growth factor $\boldsymbol{\beta}$ signaling by halofuginone. J Bioch. Mol. Biol

51. Perdereau, B. et al. Superoxide dismutase $(\mathbf{C u} / \mathbf{Z n})$ in cutaneous application in the treatment of radio-induced fibrosis. Bull. Cancer 81, 659-69

52. Lefaix, J. L. et al. Successful treatment of radiation-induced fibrosis using Cu/Zn SOD and Mn SOD: an experimental study. Int. J Radiot. Oncol. Biol. Phys 35, 305-12

53. Epperly, M. et al. Prevention of late effects of irradiation lung damage by manganese superoxide dismutase gene therapy. Gene Ther 5, 196-208

54. Kang, S. K. et al. Overexpression of extracellular superoxide dismutase protects mice from radiation-induced lung injury. Int. $J$ Radiot. Oncol. Biol. Phys 57, 1056-66

55. Vujaskovic, Z. et al. A small molecular weight catalytic metalloporphyrin antioxidant with superoxide dismutase (SOD) mimetic properties protects lung from radiation-induced injury. Free Radic. Biol. Med 33, 857-63

56. Delanian, s. et al. $\mathrm{Cu} / \mathrm{Zn}$ superoxide dismutase modulates phenotypic changes in cultured fibroblasts from human skin with chronic radiotherapy damage. Radiother. Oncol 58, 325-31

57. Benyahia. et al. Effects of superoxide dismutase topical treatment on human skin radiofibrosis: a pathological study. Breast 5, 75-81

58. Campana, F. et al. Topical superoxide dismutase reduces postirradiation breast cancer fibrosis. J Cell. Mol. Med 8, 109-116 